\title{
Creating a finite element mesh of non-periodic masonry from the measurement of its geometrical characteristics: a novel automated procedure
}

\author{
Simone Tiberti $^{1}$, Gabriele Milani $^{1}$ \\ ${ }^{1}$ Department of Architecture, Built Environment and Construction Engineering, Technical University of Milan, Piazza Leonardo da Vinci 32, \\ 20133 Milan, Italy
}

\begin{abstract}
This paper presents an automated procedure that enables the creation of a finite element mesh directly from an image file representing a rasterised sketch of a masonry element. When used to create a 2D mesh with planar and rectangular elements, the procedure is called 'pixel strategy', and the creation of a 3D mesh with elements of solid brick is called 'voxel strategy'. The homogenised in-plane and outof-plane failure surfaces of historical masonry cells that display a non-periodic arrangement of units can be extracted from the obtained finite element meshes. In our tests, these surfaces were consistent with the expected results, and their shapes suggest that the behavior of this type of masonry may range between orthotropic (if bed mortar joints are clearly noticeable) and quasi-isotropic (if some units spread over two or more masonry layers).
\end{abstract}

\section{Section: RESEARCH PAPER}

Keywords: Geometric measurement; non-periodic masonry; pixel strategy; voxel strategy; homogenisation

Citation: Simone Tiberti, Gabriele Milani, Creating a finite element mesh of non-periodic masonry from the measurement of its geometrical characteristics: a novel automated procedure, Acta IMEKO, vol. 10, no. 1, article 5, March 2021, identifier: IMEKO-ACTA-10 (2021)-01-05

Editor: Eulalia Balestrieri, University of Sannio, Italy

Received March 31, 2020; In final form August 6, 2020; Published March 2021

Copyright: This is an open-access article distributed under the terms of the Creative Commons Attribution 3.0 License, which permits unrestricted use, distribution, and reproduction in any medium, provided the original author and source are credited.

Corresponding author: Simone Tiberti, e-mail: simone.tiberti@polimi.it

\section{INTRODUCTION}

In the past 25 years, the technique known as homogenisation has emerged as one of the most reliable and effective tools for modelling the mechanical behaviour of masonry structures [1][9]. Homogenisation is a meso-scale technique using the representative elementary volume (REV) as its base. The REV is the smallest measurable volume that contains all of the physical and geometrical characteristics required for a comprehensive representation of the material. Two main modelling strategies are usually employed in applications concerning masonry: macromodelling and micro-modelling. The former considers masonry as a homogeneous material with parameters equivalent to those of the combined units and mortar [10]-[14], whereas the latter models the two constituents separately [15]-[17] and at times also considers the physical interfaces that separate them [18], [19]. Homogenisation can serve as a satisfying intermediate technique: unlike the two other strategies, it does not require experimental tests or the separate modelling of units and mortar on a large scale (this can be done at the cell level, using the REV). However, the correct geometrical representation of the masonry bond is needed for homogenisation, which can become a limiting factor when considering non-periodic masonry bonds such as are commonly found in heritage buildings. In fact, both the creation of the actual masonry geometry for meshing purposes and the generation of a finite element mesh for a suitable representation of that geometry are two significant issues that have not been addressed sufficiently in the past.

On a larger scale, historical masonry buildings present a complex geometry that must be correctly represented. Two advanced automatic survey techniques can be employed in this regard: terrestrial laser scanning (TLS) [20] and terrestrial photogrammetry [21]-[23]. These techniques are used to create finite element (FE) models from 3D point clouds [24]-[26] and have been widely applied to architectural heritage objects for purposes such as monitoring their structural health [27].

However, neither of these techniques can be used to create an FE mesh directly; instead, they are usually employed to measure the overall geometry or characterise the masonry bonds of an investigated heritage building. A review of the literature highlights the need for a simpler, faster procedure for generating 
an FE mesh using only a digital camera with suitable resolution. The input would be simply a rasterised sketch of a real masonry element, which is easy to obtain using the Image Processing Toolbox functions made available in the software MATLAB [28]. This procedure could then be implemented in a custombuilt script in MATLAB, which could be made widely available to researchers.

This paper presents two techniques for creating an FE mesh directly from the rasterised picture of a masonry element (panel, wall or pillar): one is based on the so-called 'pixel strategy' and enables the creation of a 2D FE mesh; the other creates a 3D mesh using a 'voxel strategy'. This second strategy is also extended to create a 3D FE mesh of a multi-leaf wall. The meshes obtained with these procedures are then used in numerical applications to extract homogenised in-plane and outof-plane failure surfaces, which act as macroscopic strength criteria for masonry REVs. The paper is structured as follows: Section 2 presents the automated strategies for creating the FE meshes of non-periodic masonry from the rasterised image file of the considered masonry element, for both the $2 \mathrm{D}$ and $3 \mathrm{D}$ meshes. Section 3 presents the use of the 2D FE mesh to derive in-plane homogenised failure surfaces for non-periodic masonry. Analogously, Section 4 presents the use of the 3D FE mesh to derive out-of-plane homogenised failure surfaces for nonperiodic masonry, for both single- and multi-leaf walls.
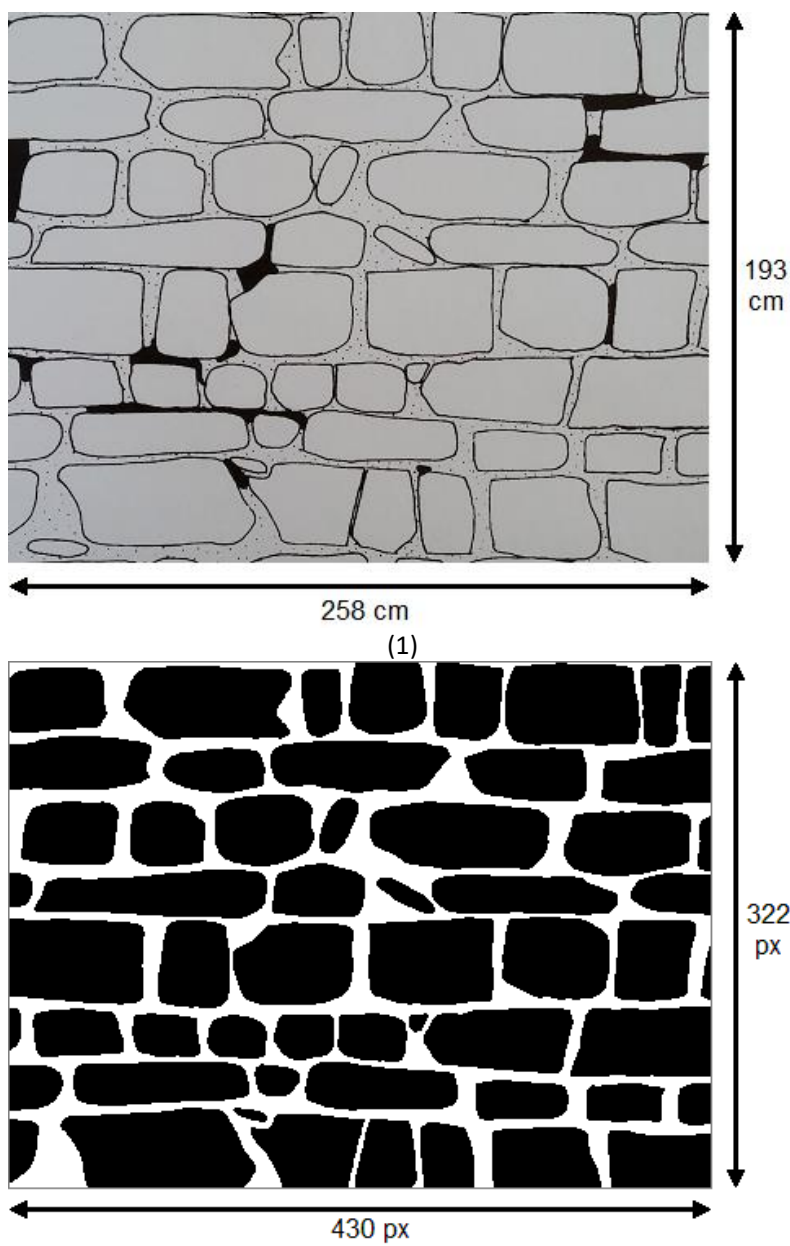

(2)

Figure 1. (1) Original redrawing of masonry panel, with dimensions in $\mathrm{cm}$ (the black parts simply denote a different mortar used in the wall), (2) rasterised sketch, with dimensions in pixel.

\section{AUTOMATED STRATEGIES FOR CREATING FINITE ELEMENT MESH OF NON-PERIODIC MASONRY}

\subsection{Pixel strategy for 2D finite element mesh}

A fast and effective procedure for the generation of an FE mesh directly from the rasterised image file of a real masonry element is presented in this section. A specific procedure called 'pixel strategy' was devised, named after the chosen procedure, in which a finite element is automatically created from one pixel of the considered rasterised image file. The rasterised image needs to be a greyscale or, preferably, a black-and-white sketch of the considered masonry bond. A dedicated MATLAB script enables the actual generation of the finite element mesh. Taking as input only the real dimensions of the masonry element under consideration, the script first extracts the red-green-blue (RGB) triplet for each pixel, which is entered into an $\mathrm{M} \times \mathrm{N} \times 3$ array $(\mathrm{M}$ and $\mathrm{N}$ are the number of pixels along the vertical and horizontal directions of the image). Second, an $\mathrm{M} \times \mathrm{N}$ matrix containing only the red values of the RGB triplet is extracted from the larger array. The red value is actually used as a threshold for determining the physical nature of the pixel (i.e. if it pertains to mortar or to a unit). Each pixel is subsequently considered to be the centroid of a planar, rectangular-shaped finite element, and the script provides it with a pair of XY coordinates that are calculated from the input global dimensions; the centre of the reference system is located at the centroid of the considered masonry element. Finally, the XY coordinates of each finite element's four adjoining nodes are determined. Overall, three matrices are thus created: one is the so-called 'node matrix', which contains the XY coordinates and the ID number of each
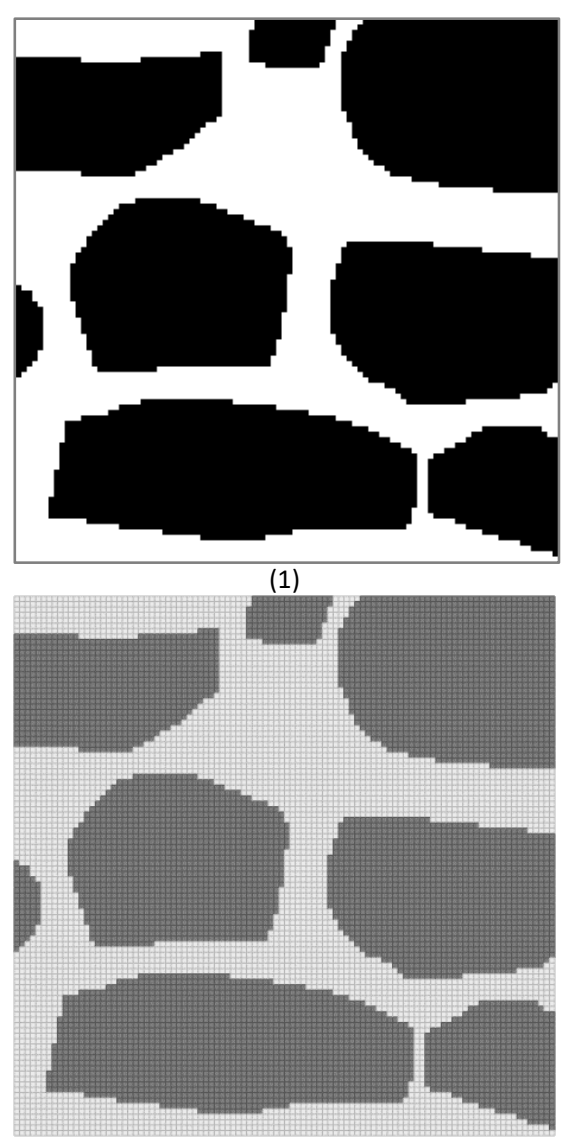

(2)

Figure 2. (1) Rasterised sketch of sample masonry panel, (2) resulting 2D finite element mesh. 
node (ordered first from top to bottom and then from left to right); the second is the so-called 'element matrix', which contains the ID number of each finite element, the ID numbers

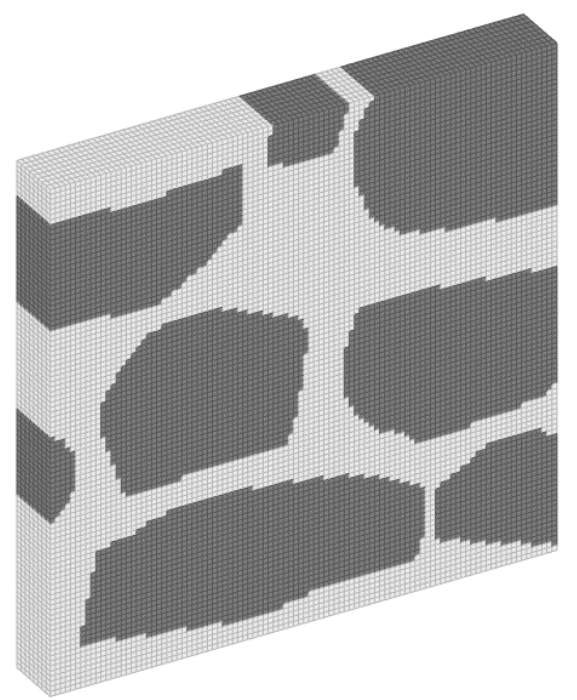

(1)

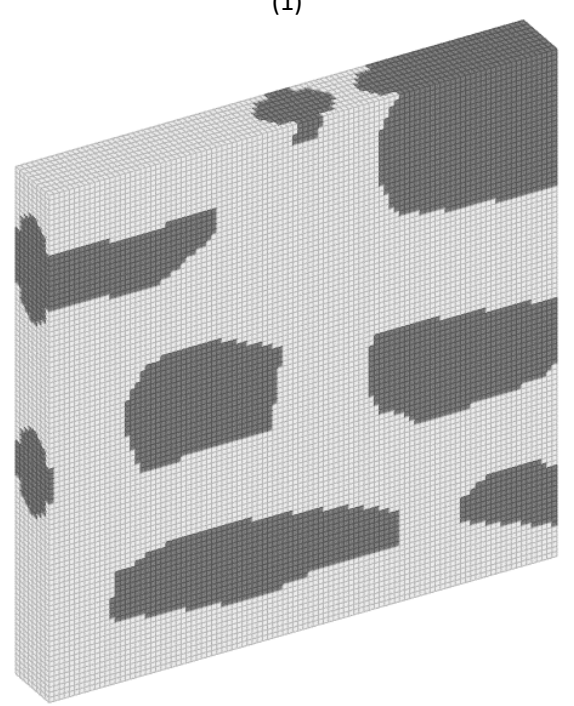

(2)

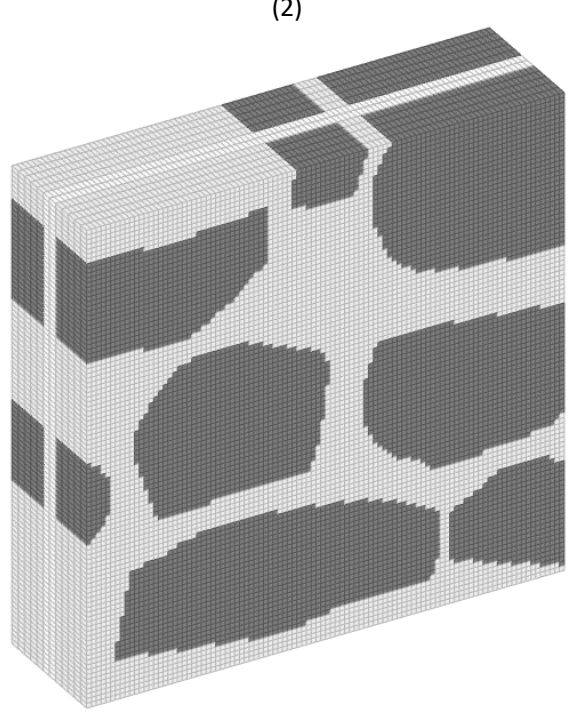

(3)

Figure 3. (1) Resulting 3D finite element mesh with extruded transversal layout, (2) resulting 3D finite element mesh with ellipsoidal stones, (3) resulting 3D finite element mesh for a multi-leaf wall. of its four nodes (listed in counterclockwise order starting from the top left corner), the XY coordinates of its centroid and finally its 'material flag', which is based on the red value of its RGB triplet. Finally, the third matrix is the so-called 'macro element matrix', which contains the ID number assigned to each unit ('macro element') in the masonry element and the XY coordinates of its centroid.

It is important to determine the correct resolution of the chosen digital camera when capturing the source image. The resolution must be adequate to accurately characterise the mortar joints so that cracks within the mortar joints are represented in the final FE mesh. Figure 1.1 shows the original redrawing of a masonry panel investigated in [29], whose dimensions are quite large $(258 \mathrm{~cm}$ in length and $193 \mathrm{~cm}$ in height). Figure 1.2 shows the rasterised sketch of the same panel, whose dimensions in pixel are 430 and 322 in length and height, respectively. The ratio between millimetres and pixels is 6:1 in this case. Hence, the resolution of this image is almost equal to 0.14 megapixels, which is enough to represent a single mortar joint with at least two pixels. Since most recent digital cameras are provided with a resolution of 20 megapixels, they should be more than sufficient for recording all of the possible geometrical features of a chosen masonry panel.

Figure 2 shows an example of the resulting finite element mesh for a sample masonry panel after the application of the pixel strategy compared to the original black-and-white rasterised source image of the panel itself.

\subsection{Voxel strategy for 3D finite element mesh}

As in the previous case, the $3 \mathrm{D}$ finite element mesh was created in MATLAB from the rasterised image file of a masonry element. Here, the mesh generation was based on the so-called 'voxel approach', in which each 3D pixel (the 'voxel') is transformed into a finite element. As above, the voxels are provided with a material flag to indicate whether they belong to a masonry unit or a mortar joint, as determined by the red value of their related RGB triplet. Because of this, units and mortar must be denoted by clearly distinguishable colours in the source image, which, again, must be a simple black-and-white or greyscale sketch of the considered masonry element. The element's overall dimensions are set as input by the user and exploited to determine the XYZ coordinates of its centroid according to a reference system originated at the centre of the test-window. This reference system is a permutation of the one created in the 2D case: here, the $y$-axis represents the horizontal axis of the test-window, the $\mathrm{z}$-axis represents the vertical axis and the $\mathrm{x}$-axis represents the transversal direction. Solid brick elements are then generated from the centroid's coordinates, and the number of elements over the transversal dimension can be set by the user. Three matrices are eventually created, one listing the node IDs and their coordinates (the 'node matrix'), the second listing the finite element IDs, those of their 8 adjoining nodes, the material flag and the coordinates of their centroid (the 'element matrix') and the third listing the macro element IDs and the coordinates of their centroid (the 'macro element matrix'). It is worth noting that the transversal layout of the considered masonry element can be derived either from the translation of its in-plane configuration or by using an ellipsoidal shape for the bricks/stones. In this latter case, the in-plane configuration of the considered masonry element denotes the mid-plane of the 3D FE mesh; the ellipsoidal shape is achieved by reducing the mid-plane surface of the bricks/stones so that their 3D shape resembles either a full ellipsoid or a truncated one. Figure 3 


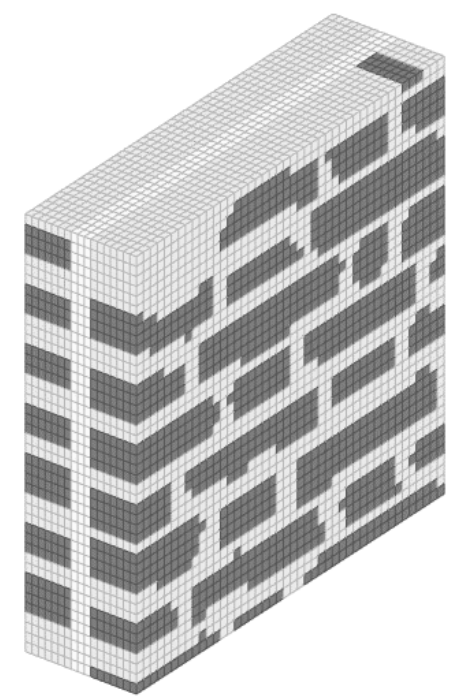

(1)
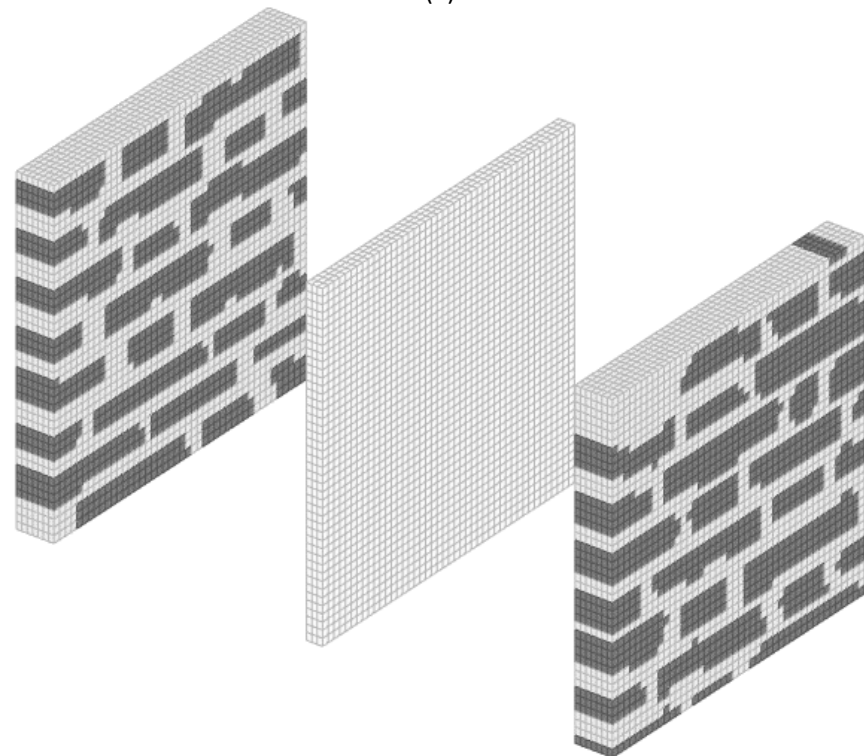

(2)

Figure 4. (1) 3D finite element mesh for a sample three-leaf masonry wall in which the two outer wythes have different bonds, (2) exploded view of the $3 \mathrm{D}$ finite element mesh highlighting the different bonds.

shows an example of the 3D meshes resulting from the application of this procedure to the sample test-window shown in Figure 2.1, including meshes obtained by using transversal extrusion (Figure 3.1) and the ellipsoidal shape of bricks/stones (Figure 3.2).

\subsection{Voxel strategy for 3D finite element mesh of multi-leaf walls}

A further MATLAB function enables the creation of a 3D FE mesh for a multi-leaf wall. The procedure is basically the same as the one for the generation of a standard 3D FE mesh, but the inputs are different: the user must choose the number of wythes for the considered multi-leaf wall and is allowed to select a separate source image for each wythe. The choice of the transversal configuration is still enabled for each wythe. Once this setup is chosen, the procedure runs in the same way as for the single-leaf 3D FE mesh. An example of the final result is shown in Figure 3.3 for a three-leaf wall in which both of the outer wythes are generated from the same source image (the testwindow in Figure 2.1) for the sake of simplicity. However, since multi-leaf masonry walls commonly display different bonds in the two outer wythes, the procedure allows the user to choose between two different rasterised sketches for the creation of the final FE mesh, as shown in Figure 4 for another sample case. It must be noted that the physical layout of the inner layer must also be correctly represented because it may consist of mortar and/or stone chips. Some transversal stones spanning the whole thickness of the wall may also be included; their presence must be carefully assessed. Of course, the layout of the inner layer cannot be achieved by simply capturing the image with a digital camera; it requires the use of more advanced techniques such as radiography or ultrasonic inspection.

\section{HOMOGENISED IN-PLANE FAILURE SURFACES}

\subsection{D finite element mesh}

The 3D FE mesh created through the procedure named 'pixel strategy' was used as input in a separate MATLAB script that was devoted to the determination of homogenised in-plane failure surfaces, which represent in-plane macroscopic strength criteria for masonry elements. They result from the solution of an upper bound limit analysis problem that includes a homogenisation approach, formulated as a minimisation problem (a sub-class of linear programming problems) in standard form. The finite elements of the mesh are supposed to be rigid; therefore, dissipation can only occur across the interfaces of adjoining elements. A Mohr-Coulomb failure criterion with a cut-off in tension is used to address the velocity jumps across the interfaces between mortar elements or between a unit and a mortar element. The equality constraints for the minimisation problem come from the velocity jumps due to dissipation, the periodicity conditions on the sides of the considered masonry element (as required by the homogenisation approach) and from the normalisation of the dissipated external power (which is needed for finding a single solution in terms of deformed configuration at collapse). Four different masonry REVs are considered in this numerical application, and they are pictured in Figure 5.1. The parameters of the Mohr-Coulomb criterion used in this application are listed in Table 1.

Figure 5.2 shows the homogenised in-plane failure surfaces in the tension-tension range for the four considered masonry REVs. It can be seen that most of them display a shape that suggests an orthotropic response under tensile loads, which is to be expected since the bed joints are clearly visible, despite a nonperiodic arrangement of the units. Only case (b) displays a quasiisotropic response, due to the presence of units that span two layers of masonry.

\subsection{D finite element mesh}

The 3D FE mesh created with the voxel strategy was similarly used to create homogenised in-plane failure surfaces as the results of another MATLAB script containing a minimisation problem. This script was modified to accept a $3 \mathrm{D}$ mesh as input instead of a 2D one. In this case, a Mohr-Coulomb failure criterion with a cut-off in both tension and compression was used to address the velocity jumps across the interfaces between

Table 1. Parameters of Mohr-Coulomb criterion for the 2D numerical application.

\begin{tabular}{cc}
\hline Mechanical parameter & Value \\
\hline Cohesion & $0.15 \mathrm{MPa}$ \\
Friction angle & $30^{\circ}$ \\
Tensile strength & $0.1 \mathrm{MPa}$ \\
\hline
\end{tabular}




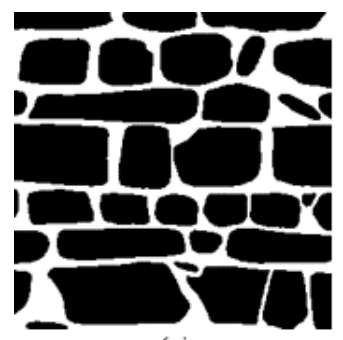

(a)

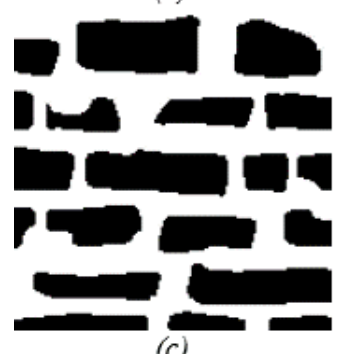

(c)

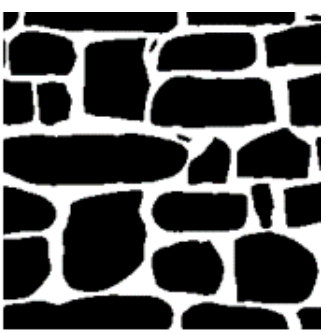

(b)

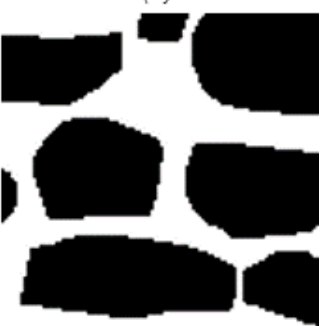

(d)

(1)

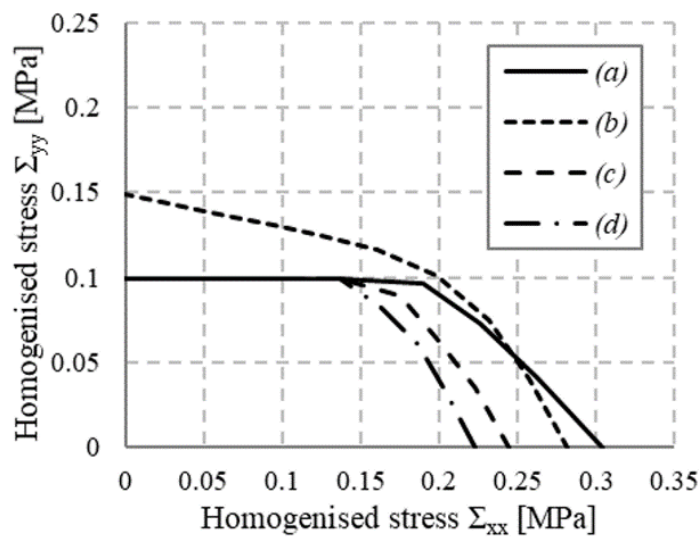

(2)

Figure 5. (1) Four masonry REVs used in the 2D numerical application, (2) comparison of the homogenised in-plane failure surfaces for the four cases.

mortar elements or between a unit and a mortar element. The parameters used in this application were those listed in Table 1 with the addition of a compressive strength equal to $1.5 \mathrm{MPa}$. Figure 6.1 shows the 3D FE mesh used in this application, which represents case (d) of the previous section. Figure 6.2 shows the full homogenised in-plane failure surface for the considered case. In the compression-compression range, the resulting homogenised failure surface is limited by the value $1.5 \mathrm{MPa}$, which is equal to the considered compressive strength; for composite in-plane load conditions, the considered masonry REV displays an increase in its strength, as expected. Figure 6.3 shows the failure modes for four uniaxial load conditions, which are all consistent with the expectations. Horizontal tension (Figure 6.3a) causes widespread cracks in the masonry testwindow, while vertical tension (Figure 6.3b) creates horizontal cracks. Horizontal compression (Figure 6.3c) also causes horizontal cracks due to lateral expansion, whereas crushing of mortar is observed in the case of vertical compression (Figure $6.3 d)$.

\section{HOMOGENISED OUT-OF-PLANE FAILURE SURFACES}

\subsection{Single-leaf wall}

Finally, the 3D FE mesh created with the voxel strategy was used to generate homogenised out-of-plane failure surfaces as the results of yet another MATLAB script containing a minimisation problem. Here, the goal was to investigate the collapse behaviour of non-periodic masonry bonds under outof-plane loads such as seismic actions.

In this case, a Mohr-Coulomb failure criterion with a cut-off in both tension and compression was used to address the velocity jumps across the interfaces between mortar elements or between a unit and a mortar element. The parameters used in this application were once again those listed in Table 1 as well as a compressive strength of 1.5 MPa. In this application, two 3D FE meshes were created for cases (b) and (c) of Figure 4, whose

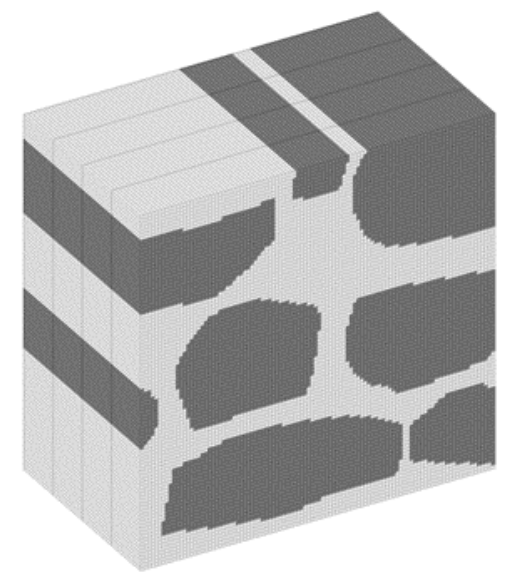

(1)

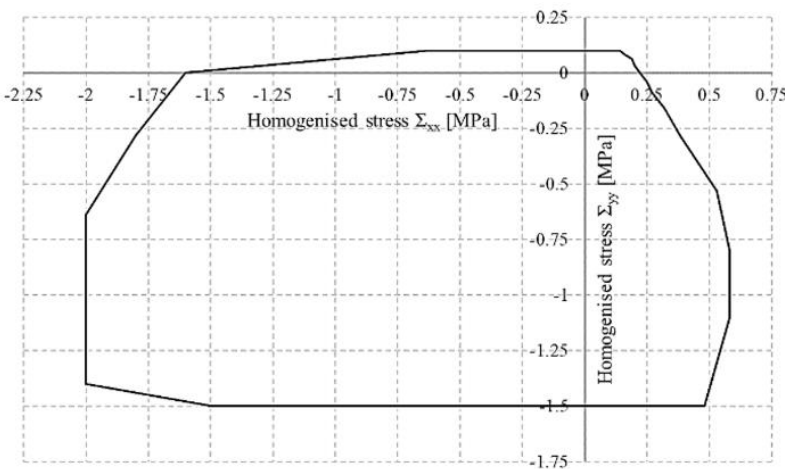

(2)

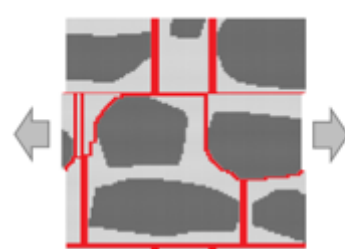

(a)

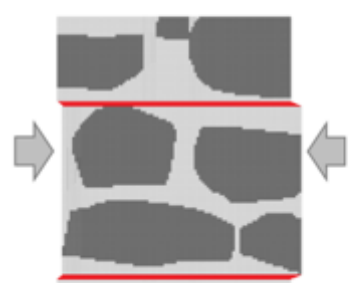

(c)

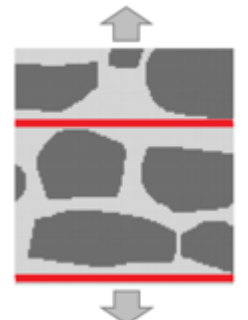

(b)

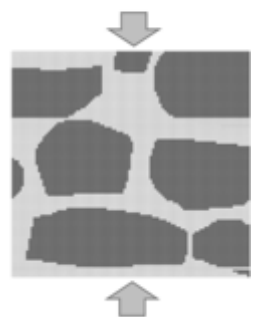

(d)
(3)

Figure 6. (1) 3D finite element mesh for case (d) of Section 3.1, (2) full homogenised in-plane failure surface for the considered masonry REV with 3D FE mesh, (3) failure modes for four uniaxial load conditions applied to the 3D FE mesh. 


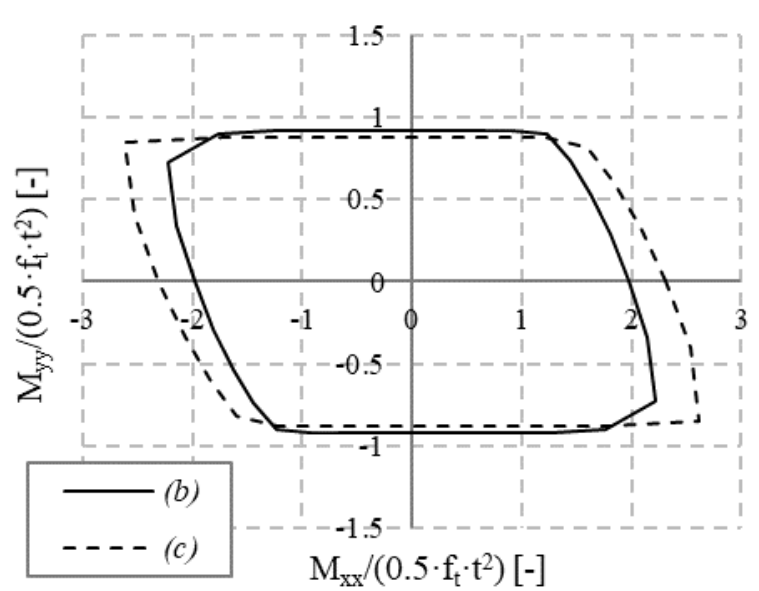

(1)

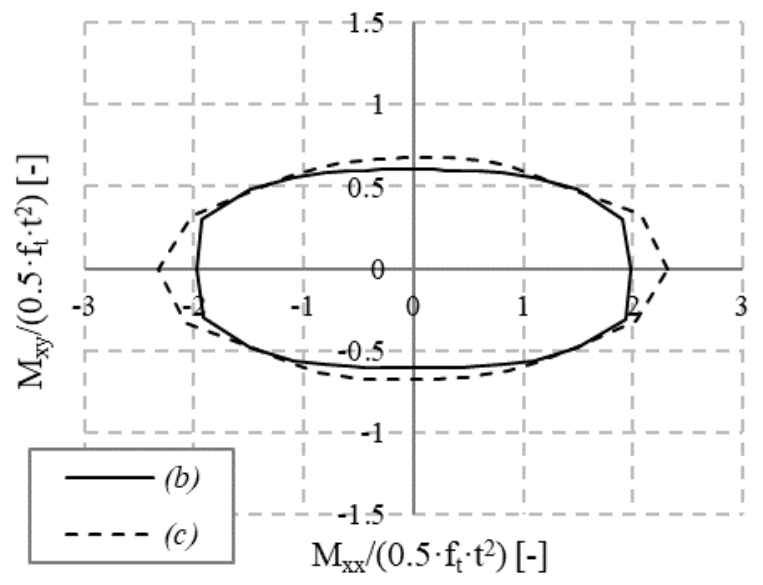

(2)

Figure 7. (1) Flexural homogenised out-of-plane failure surface for cases (b) and (c) of Figure 5 ; $(2)$ torsional homogenised out-of-plane failure surface for cases (b) and (c) of Figure 5.

depiction is omitted for sake of brevity. The thickness of the created meshes was equal to $40 \mathrm{~cm}$ for case (b) and $15 \mathrm{~cm}$ for case (c). Figure 7 shows the homogenised out-of-plane failure surfaces for the two considered cases. The aforementioned MATLAB script enables the extraction of two distinct homogenised out-of-plane failure surfaces, one dealing with the flexural collapse behaviour and defined in the $M_{\mathrm{xx}}-M_{\mathrm{yv}}$ plane and the other dealing with the torsional collapse behaviour and defined in the $M_{\mathrm{xx}}-M_{\mathrm{xy}}$ plane. $M_{\mathrm{xx}}$ is the vertical bending strength, $M_{\mathrm{yy}}$ is the horizontal bending strength and $M_{\mathrm{xy}}$ is the torsional strength. In both surfaces, the collapse moments are normalised with respect to a horizontal bending strength of $\left(f_{t} \cdot t^{2}\right) / 2$. It is possible to observe that case (c) displays a greater resistance in terms of vertical and, to a lesser extent, torsional moments. Figure 8 shows the failure modes related to case (c) extracted for three relevant out-of-plane load conditions, namely $M_{\mathrm{xx}}, M_{\mathrm{yy}}$ and $M_{\mathrm{xy}}$. They are all consistent with the expectations for a quasiregular masonry bond. Specifically, the vertical bending moment $M_{\mathrm{xx}}$ (Figure 8.1) causes widespread cracks in the masonry testwindow, while the horizontal bending moment $M_{\mathrm{yy}}$ (Figure 8.2) causes a single horizontal crack, as expected from the application of such an out-of-plane action.

\subsection{Multi-leaf wall}

As a final numerical application, the 3D mesh of a multi-leaf wall generated with the voxel strategy was used to assess the outof-plane collapse behaviour of a quasi-regular multi-leaf masonry wall. This was once again achieved by extracting homogenised out-of-plane failure surfaces that result from a modification of the out-of-plane MATLAB script described in the previous subsection. Two different instances of the considered multi-leaf wall were created: the first is depicted in Figure 9.1 and simulates the absence of mutual interaction between the three wythes (labelled ML1), whereas the second is depicted in Figure 9.2 and simulates the presence of a few transversal bricks spanning the whole thickness of the considered multi-leaf wall (labelled ML2). The first instance represents a common case observed in real multi-leaf masonry walls, in which the outer wythes are not

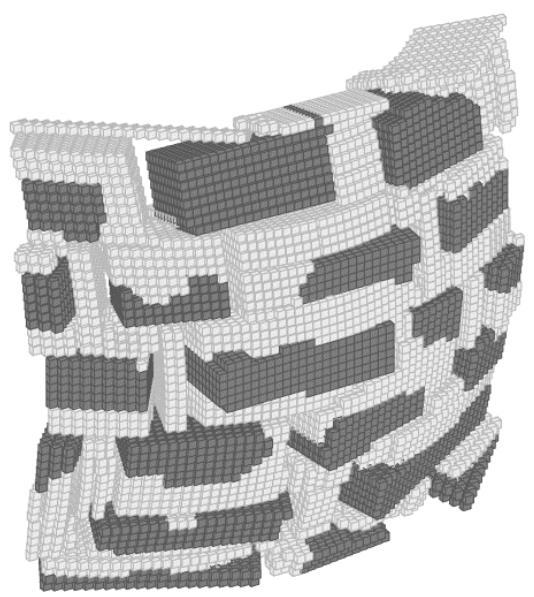

(1)

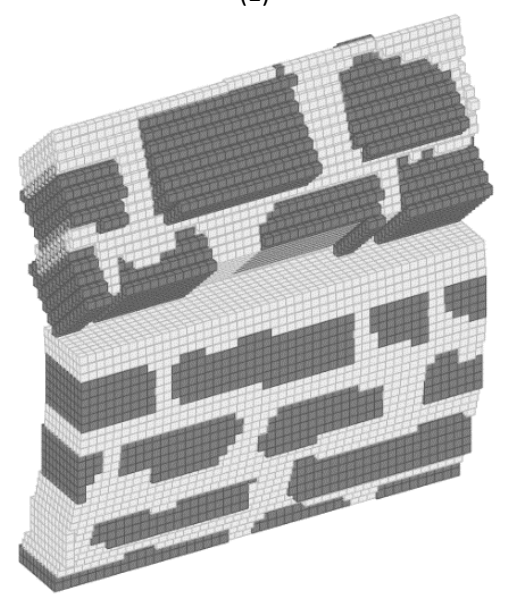

(2)

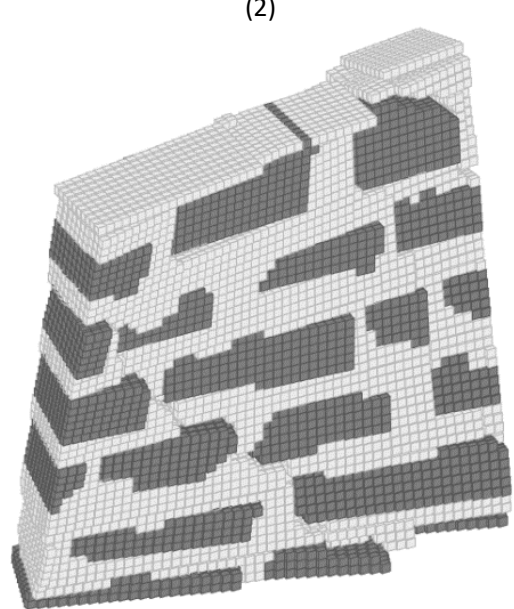

(3)

Figure 8. Failure modes for relevant out-of-plane load conditions applied to case (c): (1) application of $M x x,(2)$ application of $M y y,(3)$ application of $M x y$. 


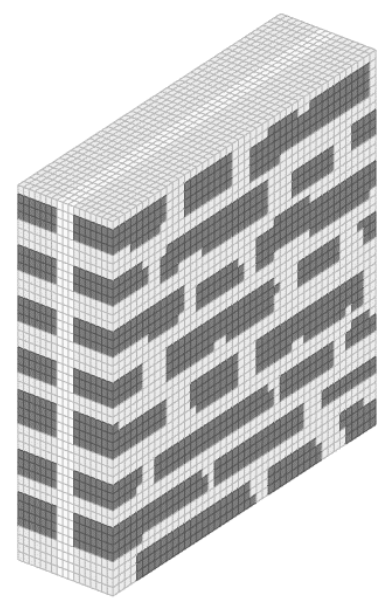

(1)
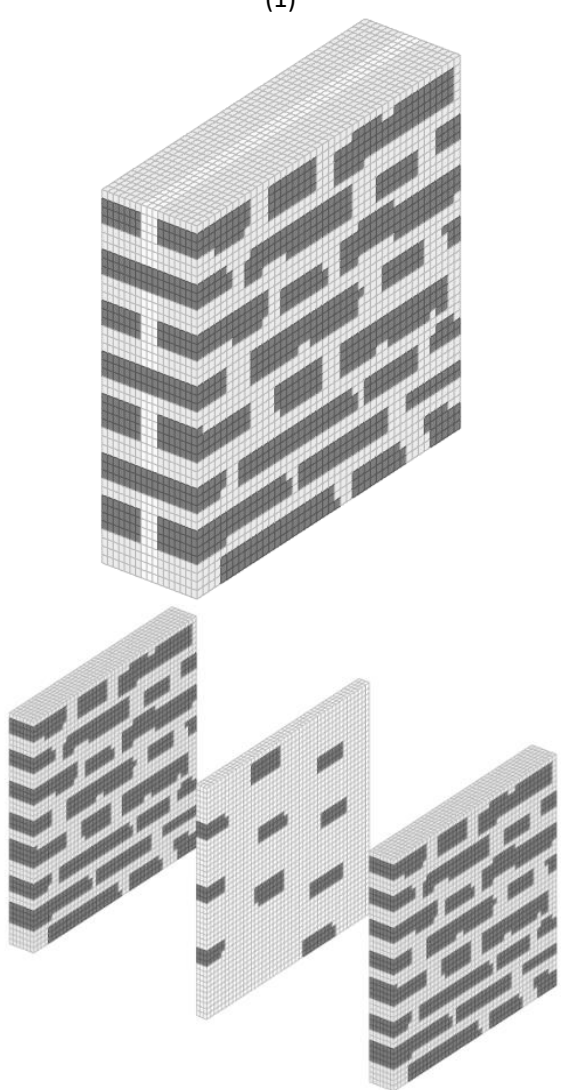

(2)

Figure 9. (1) 3D FE mesh for the instance $M L 1$ of the considered multi-lea wall, (2) 3D FE mesh for instance $M L 2$ of the considered multi-leaf wall and its exploded view to highlight the presence of transversal bricks.

connected, and the inner layer consists of loose material such as stone chips.

In each instance, the thickness of the created mesh was $36 \mathrm{~cm}$. Figure 10 presents the homogenised out-of-plane failure surfaces for the two instances of the considered multi-leaf wall. It can be observed that the instance that includes transversal bricks (ML2) shows larger homogenised failure surfaces than the instance without transversal bricks (ML1). This confirms the assumption that multi-leaf walls in which the outer wythes are somehow connected to each other (for example with transversal elements spanning the whole thickness) are more resistant to out-of-plane actions. In the case considered here, the beneficial effect of the transversal interconnection can be observed most clearly in

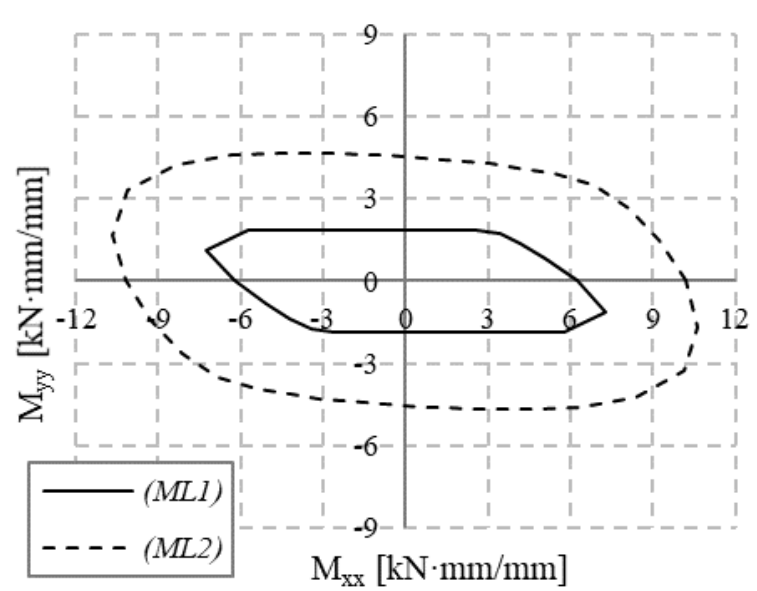

(1)

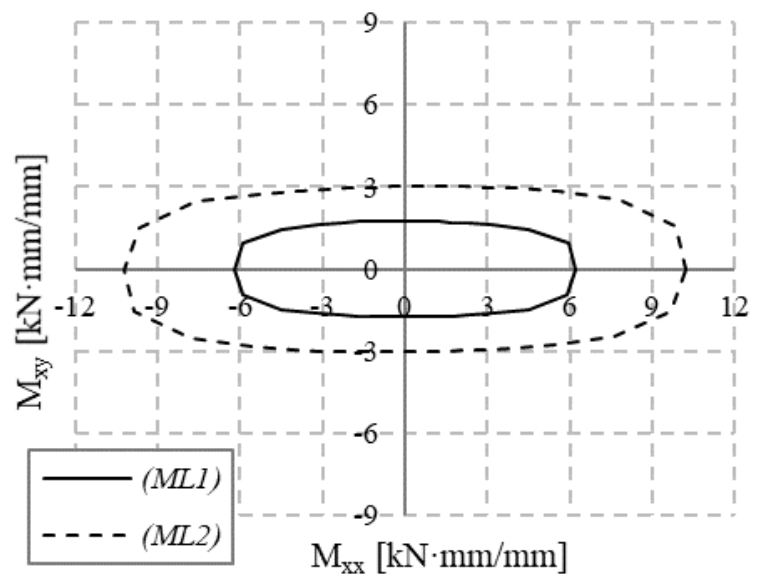

(2)

Figure 10. (1) Flexural homogenised out-of-plane failure surface for the two instances of Figure 9, (2) torsional homogenised out-of-plane failure surface for the two instances of Figure 9.

terms of resistance to $M_{\mathrm{yy}}$, which is doubled with respect to instance ML1. The resistance to $M_{\mathrm{xx}}$ and $M_{\mathrm{xy}}$ are also increased in instance ML2. Finally, Figure 11 shows the failure modes related to the two instances of the multi-leaf wall and extracted for the three relevant out-of-plane load conditions $M_{\mathrm{xx}}, M_{\mathrm{yy}}$ and $M_{\mathrm{xy}}$. Specifically, Figure 11a-c show the failure modes related to instance $M L 1$, while Figure 11d-f show the failure modes related to instance ML2. The stiffening effect created by the presence of transversal bricks is very clear for both $M_{\mathrm{xx}}$ and $M_{\mathrm{xy}}$. Furthermore, when $M_{\mathrm{yy}}$ is applied to the two instances, there is an apparent change in their failure modes; a single crack opens across a bed joint in the lower part of the multi-leaf wall in ML1, whereas there are several smaller cracks appearing across the wall in ML2.

\section{CONCLUSIONS}

A fast, automated procedure for the generation of a finite element mesh directly from the rasterised sketch of a masonry element is presented. This procedure is particularly suitable for the complex and irregular (non-periodic) masonry bonds that are often found in heritage buildings or archaeological sites. The rasterised sketch can be generated from a picture of a masonry element obtained with a digital camera with suitable resolution and MATLAB software. Two procedures were devised to create the FE mesh. One, named 'pixel strategy', converts each pixel into a single finite element, allowing for the creation of a 2D FE 


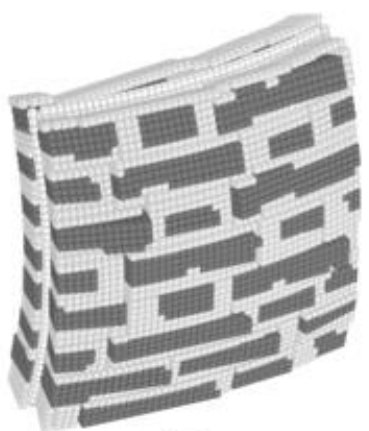

(a)

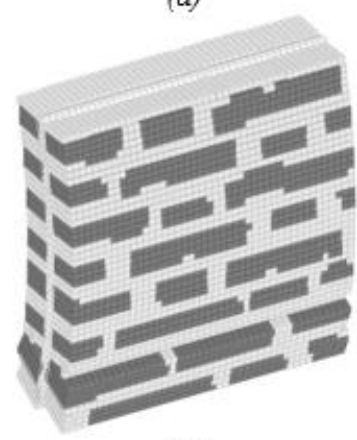

(b)

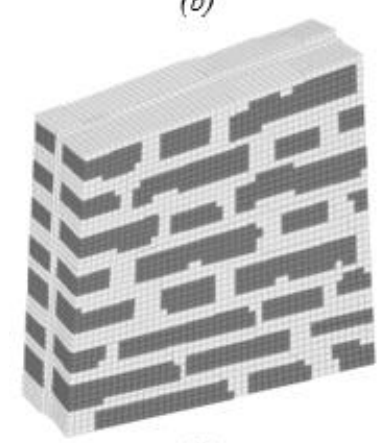

(c)

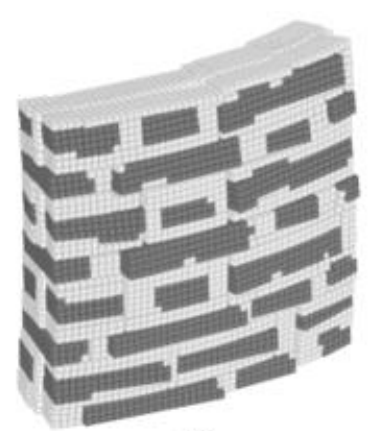

(d)

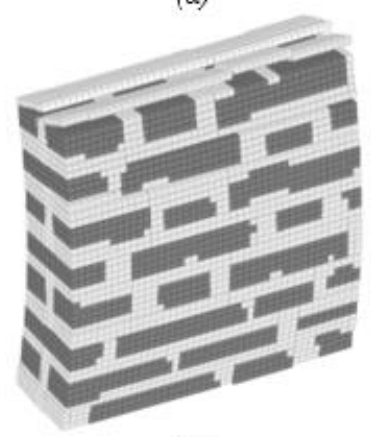

(e)

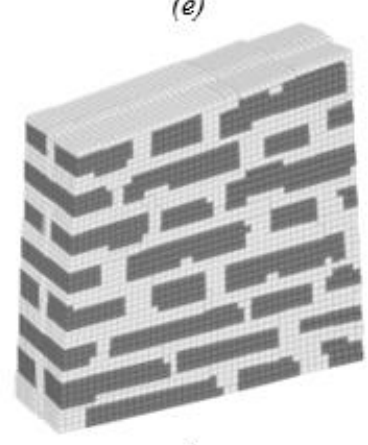

(f)
Figure 11. Failure modes for relevant out-of-plane load conditions applied to the two instances of the multi-leaf wall: $(a-c)$ instance $M L 1,(d-f)$ instance ML2.

mesh consisting of planar, rectangular elements. The other, called 'voxel strategy', first transforms a 2D pixel into an analogous 3D entity or 'voxel', which is then converted into a single finite element, enabling the creation of a 3D FE mesh consisting of solid brick elements. The 2D or 3D FE meshes can then be employed as input for several numerical applications that involve finite element analyses. In this case, the numerical applications were undertaken to extract homogenised in-plane and out-of-plane failure surfaces of masonry elements, which represent macroscopic strength criteria for in-plane and out-ofplane load conditions. Homogenised in-plane failure surfaces were derived for four masonry REVs by using 2D FE meshes developed with the pixel strategy and, in one instance, a 3D FE mesh developed with the voxel strategy. The shapes of the various surfaces indicate that non-periodic masonry displays a response that is orthotropic when the bed joints are still distinctly visible but may become quasi-isotropic in the presence of units that vertically span two (or more) masonry layers. In addition, two different sets of homogenised out-of-plane failure surfaces were extracted for two of the masonry REVs to assess in-plane collapse behaviour. Relevant failure modes were also obtained for both the in-plane and out-of-plane cases. Finally, homogenised out-of-plane failure surfaces were extracted for two instances of a quasi-regular multi-leaf masonry wall in order to assess the influence of the transversal interconnection between the outer wythes on the structural response to out-ofplane actions.

Future numerical applications will involve the implementation of the automated procedure described here in an integrated GUI-based MATLAB app that will also include a tool to rasterise a picture of a real masonry element. The proposed GUI should at least include the following features:

- A button for uploading the image of the real masonry structural element

- A window for displaying the uploaded image

- A button for starting the rasterisation procedure.

- A separate window for displaying the rasterised sketch of the source image

- A button for creating the finite element mesh and a menu that enables the selection of either a $2 \mathrm{D}$ or $3 \mathrm{D}$ mesh as well as other components (dimensions of the masonry element, etc.)

- A separate window for displaying the 2D FE mesh or the in-plane configuration of the 3D FE mesh.

- A button for starting the homogenised limit analysis of the considered test-window and a menu for selecting either in-plane or out-of-plane analysis

- A separate window for displaying the resulting in-plane or out-of-plane homogenised failure surfaces

- A button for extracting the deformed shapes at collapse and a menu for selecting the in-plane or out-of-plane load conditions for which the failure mode should be extracted

The proposed GUI-based MATLAB app could represent a useful and integrated tool for researchers and scholars who need to quickly assess the collapse behaviour of non-periodic masonry walls for academic purposes, possibly after the occurrence of a seismic sequence.

\section{REFERENCES}

[1] S. Di Nino, A. Luongo, A simple homogenized orthotropic model for in-plane analysis of regular masonry walls, Int. J. Solids Struct. 167 (2019) pp. 156-169.

DOI: $10.1016 /$ i.ijsolstr.2019.03.013

[2] G. Milani, E. Bertolesi, Quasi-analytical homogenization approach for the non-linear analysis of in-plane loaded masonry panels, Constr. Build. Mater. 146 (2017) pp. 723-743.

DOI: $10.1016 /$ i.conbuildmat.2017.04.008

[3] A. Zucchini, P. B. Lourenço, A coupled homogenisation-damage model for masonry cracking, Comput. Struct. 84 (2004) pp. 917 929.

DOI: $10.1016 /$ i.compstruc.2004.02.020

[4] A. Rekik, F. Lebon, Homogenization methods for interface modelling in damaged masonry, Adv. Eng. Softw. 46(1) (2012) pp. 35-42.

DOI: $10.1016 /$ j.advengsoft.2010.09.009

[5] [5] G. Milani, P. B. Lourenço, A. Tralli, Homogenised limit analysis of masonry walls, part I: failure surfaces, Comput. Struct. 84(3-4) (2006) pp. 166-180.

DOI: $10.1016 /$ i.compstruc. 2005.09.005

[6] G. Milani, P. B. Lourenço, Monte Carlo homogenized limit analysis model for randomly assembled blocks in-plane loaded, Comput. Mech. 46(6) (2010) pp. 827-849.

DOI: $\underline{10.1007 / \mathrm{s} 00466-010-0514-0}$

[7] G. Milani, A. Taliercio, In-plane failure surfaces for masonry with joints of finite thickness estimated by a Method of Cells-type 
approach, Comput. Struct. 150 (2015) pp. 34-51.

DOI: $10.1016 /$ j.compstruc.2014.12.007

[8] K. Sab, J. Dallot, A. Cecchi, Determination of the overall yield strength domain of out-of-plane loaded brick masonry, Int. J. Multiscale Comp. Eng. 5(2) (2007) pp. 83-92.

DOI: 10.1615/IntJMultCompEng.v5.i2.20

[9] A. Cecchi, G. Milani, A. Tralli, A Reissner-Mindlin limit analysis model for out-of-plane loaded running bond masonry walls, Int. J. Solids Struct. 44(5) (2007) pp. 1438-1460.

DOI: $10.1016 /$ j.ijsolstr.2006.06.033

[10] J. Toti, V. Gattulli, E. Sacco, Nonlocal damage propagation in the dynamics of masonry elements, Comput. Struct. 152 (2015) pp. 215-227. DOI: $10.1016 /$ i.compstruc.2015.01.011

[11] C. Gatta, D. Addessi, F. Vestroni, Static and dynamic nonlinear response of masonry walls, Int. J. Solids Struct. 155 (2018) pp. 291 303.

DOI: $10.1016 /$ j.ijsolstr.2018.07.028

[12] S. Saloustros, M. Cervera, L. Pelà, Tracking multi-directional intersecting cracks in numerical modelling of masonry shear walls under cyclic loading, Meccanica 53 (2018) pp. 1757-1776. DOI: $\underline{10.1007 / \mathrm{s} 11012-017-0712-3}$

[13] L. Miccoli, A. Garofano, P. Fontana, U. Müller, Experimental testing and finite element modelling of earth block masonry, Eng. Struct. 104 (2015) pp. 80-94.

DOI: $10.1016 /$ j.engstruct.2015.09.020

[14] F. Clementi, A. Ferrante, E. Giordano, F. Dubois, S. Lenci, Damage assessment of ancient masonry churches stroked by the Central Italy earthquakes of 2016 by the non-smooth contact dynamics method, Bull. Earthquake Eng. 18 (2020) pp. 455-486. DOI: $10.1007 /$ s10518-019-00613-4

[15] V. Sarhosis, J. V. Lemos, A detailed micro-modelling approach for the structural analysis of masonry assemblages, Comput. Struct. 206 (2018) pp. 66-81.

DOI: $10.1016 /$ i.compstruc.2018.06.003

[16] D. Baraldi, E. Reccia, A. Cecchi, In plane loaded masonry walls: DEM and FEM/DEM models. A critical review, Meccanica 53(7) (2018) pp. 1613-1628.

DOI: $10.1007 /$ s11012-017-0704-3

[17] N. A. Nodargi, C. Intrigila, P. Bisegna, A variational-based fixedpoint algorithm for the limit analysis of dry-masonry block structures with non-associative Coulomb friction, Int. J. Mech. Sci. 161 (2019) art. 105078.

DOI: $10.1016 / \mathrm{i} .1 \mathrm{ijmecsci} .2019 .105078$

[18] S. Nazir, M. Dhanasekar, A non-linear interface element model for thin layer high adhesive mortared masonry, Comput. Struct. 144 (2014) pp. 23-39.

DOI: $10.1016 /$ j.compstruc. 2014.07 .023

[19] L. Macorini, B. A. Izzuddin, A non-linear interface element for 3D mesoscale analysis of brick-masonry structures, Int. J. Numer. Meth. Eng. 85(12) (2011) pp. 1584-1608.

DOI: $\underline{10.1002 / \mathrm{nme} .3046}$
[20] J. Martínez, A. Soria-Medina, P. Arias, A. F. Buffara-Antunes, Automatic processing of Terrestrial Laser Scanning data of building façades, Automat. Constr. 22 (2012) pp. 298-305. DOI: $10.1016 /$ j.autcon.2011.09.005

[21] M. E. Stavroulaki, B. Riveiro, G. A. Drosopoulos, M. Solla, P. Koutsianitis, G. E. Stavroulakis, Modelling and strength evaluation of masonry bridges using terrestrial photogrammetry and finite elements, Adv. Eng. Softw. 101 (2016) pp. 136-148. DOI: $10.1016 /$ j.advengsoft.2015.12.007

[22] G. Milani, Y. W. Esquivel, P. B. Lourenço, B. Riveiro, D. V. Oliveira, Characterization of the response of quasi-periodic masonry: geometrical investigation, homogenization and application to the Guimarães castle, Portugal, Eng. Struct. 56 (2013) pp. 621-641.

DOI: $10.1016 /$ j.engstruct.2013.05.040

[23] S. Saloustros, L. Pelà, P. Roca, J. Portal, Numerical analysis of structural damage in the church of the Poblet Monastery, Eng. Fail. Anal. 48 (2015) pp. 41-61. DOI: $10.1016 /$ i.engfailanal.2014.10.015

[24] A. Guarnieri, F. Pirotti, M. Pontin, A. Vettore, Combined 3D surveying techniques for structural analysis applications, Int. Arch. Photogrammetry Remote Sens. Spatial Inf. Sci. 36(6) (2005). Online [Accessed 21 March 2021] https://www.isprs.org/proceedings/xxxvi/5-W17/pdf/28.pdf

[25] A. Guarnieri, N. Milan, A. Vettore, Monitoring of complex structure for structural control using Terrestrial Laser Scanning (Tls) and photogrammetry, Int. J. Archit. Heritage 7 (2013) pp. 5467. DOI: $10.1080 / 15583058.2011 .606595$

[26] I. Lubowiecka, J. Armesto, P. Arias, H. Lorenzo, Historic bridge modelling using laser scanning, ground penetrating radar and finite element methods in the context of structural dynamics, Eng. Struct. 31(1) (2009) pp. 2667-2676. DOI: $10.1016 /$ i.engstruct.2009.06.018

[27] C.-H. Wang, J. P. Mills, P. E. Miller, Automated low-cost photogrammetry for flexible structure monitoring, Int. Arch. Photogrammetry Remote Sens. Spatial Inf. Sci. 39 (2012) pp. 393 398. Online [Accessed 21 March 2021] https://www.photomodeler.com/downloads/documents/applic ations/ISPRS-2012-Monitor-Flexible-Structures.pdf

[28] MATLAB Release 2018b, The MathWorks, Inc., Natick, Massachusetts, United States.

[29] Ministero per i Beni Culturali e Ambientali. Atlante dei tipi costruttivi murari Italia Settentrionale. Schedatura tecniche murarie. Area $n^{\circ} 13$. Valli di Parma e Reggio. Online [Accessed 21 March 2021]

http://www.culturaitalia.it/opencms/viewItem.jsp?language=it\& $\mathrm{id}=$ oai $\% 3$ Awww.michael-culture. $i t \% 3$ Apubit $\% 2$ Fdcollection $\% 2$ FIT-DC-76630c5f 